\title{
EL MODELO SUECO DE CARRERA DOCENTE E INVESTIGADORA
}

\section{THE SWEDISH MODEL OF TEACHING AND RESEARCH CAREER}

\author{
AUTORES \\ Dr. Carlos P. de los Heros: Department of High Energy Physics \\ cph@tsl.uu.se
}

Box 535. Uppsala University

tel:+4618 4713256 S-75121 fax:+46 184713513

Uppsala. Sweden

\section{CURRÍCULUM VITAE}

Físico nuclear, doctorado en Israel y afincado en Suecia, investiga para EE.UU. en un proyecto que busca descubrir la cuarta dimensión.

\section{RESUMEN}

Es necesario hacer unas aclaraciones previas. En lo que sigue me referiré frecuentemente al NFR (Secretaría para la Investigación). Es éste un órgano, formado por científicos, dependiente del Ministerio de Educación y Ciencia sueco, que lleva casi todo el peso de la financiación de la investigación en todos los campos. El órgano rector se elige democráticamente entre los profesores universitarios, por lo cual su composición no esta ligada al partido en el poder en cada momento. 
Modelo sueco - Docencia - Investigación

\begin{abstract}
It is necessary to make some preliminary clarifications. In what follows I will refer frequently to the NFR (Ministry for Research). This is a body consisting of scientists, under the Ministry of Education and Science Sweden, which has almost the entire burden of financing of research in all fields. The governing body is elected democratically from among university professors, so their composition is not linked to the party in power at any time.
\end{abstract}

\title{
KEY WORDS
}

Swedish model - Teaching - Research

Tras el debate abierto, hace meses, en la lista de distribución de la AACTE, sobre los modelos de carrera universitaria en países extranjeros, he recopilado esta información referente al modelo sueco, que me agrada haga pública la Redacción de Vivat Academia. Si alguno de los lectores necesita ampliación de lo aquí escrito, no existe inconveniente alguno en que se ponga en contacto personalmente conmigo en la siguiente dirección

Asimismo, si hubiere alguna información adicional a tener en cuenta, no duden en hacérmela llegar, bien directamente, bien a través de la revista 
Es necesario hacer unas aclaraciones previas. En lo que sigue me referiré frecuentemente al NFR (Secretaría para la Investigación). Es éste un órgano, formado por científicos, dependiente del Ministerio de Educación y Ciencia sueco, que lleva casi todo el peso de la financiación de la investigación en todos los campos. El órgano rector se elige democráticamente entre los profesores universitarios, por lo cual su composición no esta ligada al partido en el poder en cada momento.

El órgano rector nombra subcomités, de tres años de duración, para las distintas áreas. Los miembros de los subcomités ya no están elegidos democráticamente, sino basándose en su prestigio en el campo relevante. Al cabo de ese tiempo el órgano rector elige nuevos miembros. Se suele tender a mantener un equilibrio regional, de modo que los miembros de los subcomités provengan de todo el territorio nacional. Se puede ser reelegido para un subcomité por un segundo periodo de 3 años, pero no más en forma continua. Los subcomités se encargan de tomar decisiones de política científica, (o bien hacer recomendaciones a instancias más altas del gobierno), de hacer el seguimiento de los proyectos y de supervisar la selección de candidatos para plazas universitarias.

Por lo que se refiere a la categoría administrativa de los puestos docenteinvestigadores debemos decir que no son funcionariales, en el sentido español del término. Es decir, no se trata de puestos gubernamentales. Cada Universidad tiene libertad para establecer su rango salarial, de modo que el mismo puesto puede tener diferentes salarios en diferentes centros superiores.

Los tipos de plazas son fundamentalmente cuatro: Postdoc, Investigador Asociado (Research Assistant), Titular (University Lecturer) y Catedrático (Professor). Existe un quinto tipo de plaza "tenure track", de carácter extraordinario, de la que hablaremos al final de este artículo. 
Los dos primeros y el último son puestos temporales, los dos intermedios son fijos. El "Research Assistant" inicia el camino hacia la Titularidad (así como la "tenure track"). La primera criba se realiza en el paso de Postdoc a Research Assistant. Aquél que no consigue una plaza de tipo Investigador Asociado está prácticamente fuera de la carrera universitaria.

A continuación daremos un pequeño repaso a las particularidades de estos puestos de trabajo:

\section{Postdocs:}

Duración: 2 años. Se requiere, obviamente, tener el título de doctor. El Investigador Principal (IP) de cada proyecto solicita directamente al NFR la financiación para mantener el número de Postdocs necesarios. La Facultad y la Universidad no se ven involucradas en este proceso. Es el subcomité relevante, correspondiente al campo de investigación del IP en el NFR, el que decide sobre la financiación, basado en los méritos del IP y el proyecto para el cual pide el Postdoc. Si el NFR proporciona el dinero, entonces el IP hace pública la convocatoria de la plaza y es él mismo el que se encarga de la selección de candidatos. Dónde se publica la plaza y los baremos de selección dependen entonces únicamente del IP. Pero hay que tener en cuenta que al IP le interesa mantener un grupo bueno de investigación porque si no se la juega en futuras peticiones de dinero al NFR (ver la sección sobre profesores Titulares mas abajo).

En rarísimas ocasiones es la Facultad la que proporciona el dinero para mantener un Postdoc, pero es una excepción posible. La financiación para estos puestos proviene casi exclusivamente del NFR. 
No se le requieren al candidato traducciones ni homologaciones de títulos, se evalúa su curriculum vitae, la lista de publicaciones y posiblemente cartas de recomendación (ver mas abajo).

Investigador Asociado:

Duración: 2+2 años. Es un puesto dedicado únicamente a la investigación, sin responsabilidad docente alguna. Al cabo de los dos primeros años el trabajo del candidato es evaluado antes de prorrogarlo por los otros dos siguientes. Al cabo de 4 años no hay garantía de promoción al nivel siguiente. Si la Facultad así lo decide, sacará una plaza de Titular por la que el Investigador Asociado deberá competir con posibles candidatos externos.

Hay dos formas de acceder a este tipo de puesto:

i) Un grupo, a través de su Investigador Principal, propone a la Facultad correspondiente la creación de una plaza de Investigador Asociado. El Decanato ha de aprobarlo antes de realizar la propuesta al Rectorado de la Universidad. Si éste lo aprueba, la Facultad entonces queda encargada de anunciar la plaza y cubrirla. Al menos en Física, los anuncios se hacen en revistas y publicaciones internacionales. Sin embargo, la decisión de cómo proceder a cubrir la plaza depende de la Facultad convocante.

ii) Un individuo puede solicitar directamente al NFR una plaza de Investigador Asociado, sin que medie un grupo de investigación. El NFR evalúa sus méritos académicos y, si decide darle la plaza, el candidato entonces elige a qué Universidad incorporarse, con el acuerdo de ésta, por supuesto. Existen dos convocatorias anuales para este tipo de vía.

Un Investigador Asociado puede formar su propio grupo (sobre todo si accede al puesto a través del programa directo del NFR). Maneja presupuesto para su 
investigación y puede dirigir tesisNOTA. En principio, como hemos mencionado, no tiene carga lectiva alguna, aunque puede dedicar hasta un 20\% del tiempo a la enseñanza. En este caso la duración del contrato es de 2+3 años.

Se accede a este tipo de puesto únicamente por méritos. Hay que rellenar un formulario, (en el caso del NFR al menos, no estoy seguro de si lo mismo ocurre cuando es la Facultad la convocante. de la plaza), presentar el curriculum vitae, la lista de publicaciones y, algo muy importante, un proyecto de investigación. Se pueden adjuntar cartas de recomendación, pero no es requerido formalmente. Esto es debido a que en Suecia toda la documentación que uno presenta a la administración, se convierte automáticamente en un documento público, de modo que el valor de una carta de recomendación queda algo desvirtuado si el que la escribe sabe que puede ser pública.

La selección del candidato se lleva a cabo por el subcomité relevante del NFR o por un 'comité de selección', en el caso de que sea la Facultad la que saque a concurso la plaza. Estos comités suelen ponerse en contacto con un experto del campo de que se trate, (normalmente un experto nacional para este tipo de plazas), para que evalúe a los candidatos. Este experto hace una recomendación al comité que lleva la selección, en la que propone 2 ó 3 nombres entre la lista de candidatos. Los candidatos preseleccionados han de dar una clase a nivel de primer ciclo sobre un tema propuesto por el comité (el mismo tema para todos los candidatos). También hay una sesión de preguntas a los postulantes sobre su actividad investigadora.

En el caso de que se trate de una plaza del NFR, el subcomité decide. En el caso de que sea la Facultad, el comité de selección no decide, sino que con la información proporcionada por el experto, más su propia evaluación, hace una recomendación al Decanato, quien tiene la última palabra sobre la contratación. Normalmente 
simplemente toman la recomendación del comité tal y como viene. Pero legalmente pueden cambiarla.

Titulares:

Y aquí viene una de las más sorprendentes características del sistema sueco. Son plazas creadas por la Universidad, ÚNICA y EXCLUSIVAMENTE para enseñar y, en principio, abierta según las necesidades lectivas de la Facultad correspondiente. Dense ustedes cuenta que éste es el primer y único nivel permanente (a excepción de la Cátedra) en el sistema y que en los niveles anteriores no se prima la docencia en absoluto.

Si uno quiere dedicarse a la investigación, tiene que pedir fondos (grants) para ello al NFR, o a los fondos especiales que cada universidad tiene para estos fines. Si el proyecto es financiado, entonces el profesor es relevado de un porcentaje del tiempo de enseñanza. Este porcentaje de 'liberación de tareas de enseñanza' no puede pasar del $75 \%$. Lo normal es obtener un $50 \%$.

Para poder obtener financiación para un proyecto, el candidato, (que, recuerdo, a este nivel ya tiene su puesto fijo como profesor de universidad con un $100 \%$ de su tiempo dedicado, en principio, a la enseñanza), ha de presentar un proyecto y su curriculum vitae con lista de publicaciones, por supuesto. Si la petición de financiación va al NFR (lo cual es habitual), es el subcomité del campo relevante el que decide. Si la petición de fondos se hace a la Universidad, entonces es la Facultad la que decide. Este tipo de petición no es de tipo competitivo, es decir, con otros candidatos en el mismo proyecto. Lo que tiene que ser convincente es el proyecto y la trayectoria del individuo y su grupo.

En caso de obtener financiación, ésta es por tres años. Al cabo de esos tres años se tiene que volver a pedir la exención del porcentaje correspondiente de la enseñanza, 
mediante la presentación de un nuevo proyecto, o la petición de re-evaluación para la continuación del que ya se tiene. En el caso que se obtenga financiación para investigar, entonces se pueden dirigir tesis doctorales. Si no, el puesto en sí es para la enseñanza a nivel de licenciatura ( $1^{\circ}$ y $2^{\circ}$ ciclos). Así que para mantener la investigación, la actividad de cada profesor universitario es reevaluada cada tres años. En casos de inoperancia patente se puede perder la financiación para investigar, (y para mantener un grupo), quedando únicamente dedicado a la enseñanza a nivel de licenciatura; al menos por tres años, hasta la siguiente oportunidad para pedir dinero de nuevo...Pero como una de las cosas a evaluar es la trayectoria investigadora del candidato, el haber estado en el 'dique seco' durante tres años, sin publicaciones ni contacto directo con doctorandos, y el mundillo correspondiente, puede afectar negativamente a las posibilidades de obtener presupuesto de nuevo. En resumen, uno 'compra' tiempo de investigación con su curriculum y su trayectoria investigadora.

\section{Catedráticos:}

Puestos creados por la Universidad debido a que una plaza ya existente queda vacante, o porque hay necesidad de cubrir o iniciar un nuevo campo de investigación. Tienen entre un 10 y 20\% del tiempo dedicado a la enseñanza de primer ciclo. Y se supone que han de participar en el segundo ciclo y en la dirección de tesis doctorales, pero esto último no está legalmente requerido. Si lo desean, pueden reducir sus actividades docentes a ese mínimo del 10\% en el primer ciclo. Es una plaza permanente y no hay evaluación de las actividades que realiza.

La plaza se cubre tras un anuncio en revistas especializadas de ámbito internacional. La Universidad elige tres expertos internacionales de renombre en el campo correspondiente para que evalúen el curriculum vitae y la trayectoria investigadora de los candidatos. Este trío de expertos produce una lista en donde se ordenan los candidatos de acuerdo con sus méritos. Esta es su recomendación a la Universidad, 
que a la vez la pasa al Ministerio, ya que el nombramiento final de catedrático es competencia de éste último.

Actualmente los catedráticos son empleados por la Universidad, no por el Estado como sucedía hace algunos años.

Últimamente ha surgido una nueva 'clase' de catedráticos: los profesores Titulares que tengan méritos suficientes pueden pedir el título de Catedrático. Se trata simplemente del título, que puede añadir a su nombre, no de los privilegios (libertad para enseñar o no, mayor poder de decisión en asuntos de la Facultad etc.) que tienen los catedráticos que acceden por el sistema mencionado más arriba. Esto se introdujo para equiparar un poco el sistema de títulos con el anglosajón, ya que en el modelo sueco a Catedrático no llega cualquiera simplemente por edad o méritos, sino que esta ligado también a la existencia de plazas libres. Y el número de plazas de Catedrático es mas bien fijo, no se crean alegremente. Se daba así el caso de profesores Titulares con experiencia, prestigio y edad suficientes para ser catedráticos, y que en los Estados Unidos de América serían 'Professors'. Para evitar agravios comparativos en reuniones o comités internacionales, se ha creado este nuevo tipo de Catedrático. Como mencionaba anteriormente, se posee el título, pero las actividades diarias, deberes, derechos y sueldo son los mismos que los del profesor Titular. Obviamente han habido algunas voces en contra de este método, pero de momento se ha implantado.

Tenure Tracks:

Este quinto tipo extraordinario de plazas, muy a semejanza de los americanos, funciona de la siguiente manera: Son convocadas por el NFR para un campo de investigación concreto. Las convocatorias son extraordinarias, ya que salen cuando se quiere potenciar un tema de investigación o iniciar un campo nuevo. Eso quiere decir que no son convocadas cada año junto con el resto. En la última convocatoria salieron 
siete plazas para temas que van desde Biología a Estadística pasando por Física. La convocatoria puede llegar a ser bastante específica, incluso para un experimento concreto. He aquí un ejemplo de convocatoria real: "Experimental Astroparticle physics related to the Amanda experiment".

Para concursar hay que presentar el curriculum vitae, (con la consabida lista de publicaciones, con una copia de hasta 10 de las que uno considere más relevantes), y el proyecto de investigación. También se pueden presentar otro tipo de méritos, como dice la convocatoria: "Other documents that the candidate wants to refer to could also be included", por ejemplo, cartas de recomendación. El NFR elige entonces tres personas de renombre internacional en el campo de que se trate, para que evalúen la documentación de los candidatos y hagan su recomendación. Estos 'jueces' son extranjeros. El comité relevante del NFR normalmente toma la recomendación de los jueces y selecciona al candidato sugerido por ellos. Hasta el momento la Universidad no ha jugado papel alguno. Es el candidato seleccionado el que elige a qué Universidad y Departamento incorporarse, con el visto bueno del Departamento, por supuesto. La Universidad, al aceptar al candidato con este tipo de plaza, se compromete a convertir en fija la plaza al cabo de 6 años (una "University Lecturer", es decir, profesor Titular). El NFR subvenciona totalmente el salario y gastos de la plaza por los 6 primeros años. A partir de ese tiempo, la Universidad corre con los gastos.

Hay una evaluación de la investigación realizada al cabo de los 3 primeros años. En ese momento es realmente cuando la Universidad se tiene que comprometer a hacer la plaza fija al cabo de otros 3 años más. Si el interesado no ha rendido satisfactoriamente en su investigación o tareas docentes, esa es la ocasión en la que puede perder el puesto de trabajo. Durante los 6 primeros años pagados por el NFR, hay un porcentaje del tiempo que ha de dedicarse a la enseñanza, a negociar entre candidato y departamento. El NFR recomienda 75 horas de docencia al año. Al cabo 
de los 6 años, cuando se pasa a ser un "University Lecturer" como otro cualquiera, entonces ya asume la docencia típica de ese tipo de puestos.

La idea de estas plazas es atraer a gente buena en campos que interesan en Suecia. Se supone entonces que un buen candidato elegirá un buen Departamento, y que el Departamento estará contento de recibir un buen candidato. De modo que ambos, (y el sistema en último término), se benefician. Hay que tener en cuenta que el Departamento no ha participado, en absoluto, en el proceso de selección, aunque siempre se puede negar a acoger al interesado, y entonces éste tiene que "buscar" otra Universidad/Departamento que le acepte. La verdad es que no conozco casos de rechazo de candidatos en este tipo de plazas.

\section{GENERALIDADES:}

Todo el proceso de selección es completamente gratuito para los candidatos. No se requiere documentación compulsada alguna, (se admiten simples fotocopias), ni títulos homologados, ni fotocopias de documentos de identidad. En general, los impresos a rellenar son fácilmente accesibles en la página Internet del NFR o de la Facultad correspondiente.

Toda la documentación entregada al NFR, o a la Facultad, para participar en el concurso a una plaza es automáticamente publica, de modo que los otros candidatos (o quién sea) puedan tener acceso a ella.

El sistema no prevé la existencia del 'año sabático'. Se puede negociar con la Facultad a título individual.

No hay ningún requerimiento de nacionalidad, ni siquiera el pertenecer al ámbito de la UE. En principio, tampoco sobre el dominio del idioma. Por supuesto se espera 
que el candidato seleccionado sea capaz de enseñar en sueco al cabo de algún tiempo.

NOTA: Un "Research Assistant" puede efectivamente dirigir doctorandos, pero sólo si éstos se incorporan al proyecto prácticamente al mismo tiempo que él, ya que su puesto es de una duración máxima de cuatro años. Por tanto, sólo en este caso podría dirigir la correspondiente tesis doctoral durante todo el periodo que dura el programa de doctorado en Suecia (4 años). Si no, para el caso de estudiantes de doctorado que se incorporen al proyecto en otro momento, el Investigador Asociado puede actuar como co-director, pero no como único director, para evitar así que, si al cabo de su contrato no obtiene la Titularidad, el doctorando se quede sin supervisor. La Facultad se encarga de organizar las cosas para que no surjan este tipo de problemas y que un doctorando siempre tenga un director titular en caso de conflicto. 